\title{
Effects of Thickness and Crystallographic Orientation on Fatigue Life of Single-Crystalline Copper Foils
}

\author{
Kazuki Kammuri ${ }^{1, * 1}$, Masashi Kitamura ${ }^{1, * 2}$, Toshiyuki Fujii ${ }^{2, * 3}$ and Masaharu Kato ${ }^{3}$ \\ ${ }^{1}$ Department of Innovative and Engineered Materials, Tokyo Institute of Technology, Yokohama 226-8502, Japan \\ ${ }^{2}$ Department of Metallurgy and Ceramics Science, Tokyo Institute of Technology, Tokyo 152-8552, Japan \\ ${ }^{3}$ Department of Materials Science and Engineering, Tokyo Institute of Technology, Yokohama 226-8502, Japan
}

\begin{abstract}
Thickness effect on fatigue life of pure copper single-crystal foils with two different surface orientations, $(21 \overline{1})$ and $(5 \overline{11} \overline{1})$, but with the same stress-axis orientation was investigated by means of $S-N$ curve measurement and microstructural observation. Thicknesses of the foils were

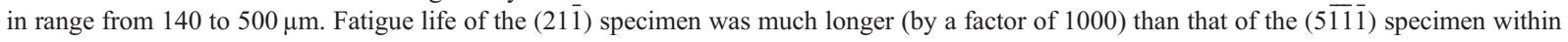
the given range of the foil thickness. The orientation dependence of fatigue life became more pronounced with decreasing the foil thickness. The size and crystal orientation dependence of the fatigue life can be explained reasonably by considering the reduction of the net area of the primary and secondary slip planes during deformation. [doi:10.2320/matertrans.M2014352]
\end{abstract}

(Received October 2, 2014; Accepted November 14, 2014; Published December 27, 2014)

Keywords: copper foil, fatigue, single crystal, crystallographic orientation, slip system

\section{Introduction}

The mechanical behavior of the thin metal foils is a key factor determining the long-term service life of MEMS (micro-electromechanical system). Further, fatigue behavior of copper foils has attracted much attention as they are used widely in flexible printed circuits. ${ }^{1,2)}$ Recently, thin copper foils are being employed as fine pitch wiring in highly miniaturized devices. Under these circumstances, a number of studies have been conducted to investigate the so-called size effect on mechanical properties of thin sheet metals with the thicknesses ranging from about $200 \mathrm{~nm}$ to $500 \mu \mathrm{m} .^{3-17)}$

It is well known that the mechanical strength of metallic thin foils exceeds those of bulk specimens. To understand the underlying mechanism reasonable for this phenomenon, several models ${ }^{3-7)}$ have been developed, including the dislocation starvation model ${ }^{5)}$ and strain gradient plasticity models. ${ }^{5,6)}$ Hommel et al. $^{7)}$ reported the stress-strain behavior of thin foils $(0.4-3.2 \mu \mathrm{m})$. Motz et al. ${ }^{6)}$ showed that the increase in the flow stress of thin foils becomes significant when the foil thickness becomes less than $5 \mu \mathrm{m}$, while a weak increase in the flow stress is observed with decreasing foil thickness to $5 \mu \mathrm{m}$ in their bending tests.

In the case of fatigue, it has also been reported that fatigue strength increases as the thickness of a specimen decreases to a few microns. Kraft et $a l .{ }^{8)}$ showed that thinner copper film specimens (with thickness of $0.3-3.1 \mu \mathrm{m}$ ) on polyimide substrates have longer fatigue lives. Zhang et al. ${ }^{9)}$ investigated surface damage and dislocation structure after cyclic deformation of copper films with different thicknesses $(0.2-$ $0.3 \mu \mathrm{m})$ and grain sizes $(0.3-2.1 \mu \mathrm{m})$. They found that fatigue damage and dislocation structure were determined not only by foil thickness but also by grain size. The size effect in films thinner than $3 \mu \mathrm{m}$ was explained on the basis of the disappearance of fatigue dislocation structures and the

\footnotetext{
${ }^{* 1}$ Graduate Student, Tokyo Institute of Technology

${ }^{* 2}$ Graduate Student, Tokyo Institute of Technology. Present address: Mitsubishi Heavy Industries, Ltd., Takasago 676-8686, Japan

${ }^{* 3}$ Corresponding author, E-mail: fujii.t.af@m.titech.ac.jp
}

decrease of the dimensions of extrusions. ${ }^{9,10)}$ In the case of films with dimension of approximately $20-500 \mu \mathrm{m}$, Judelewicz et al. ${ }^{11)}$ reported that a thinner film exhibits a longer fatigue life in free-standing copper foils with the thickness ranging from $20 \mu \mathrm{m}$ to $300 \mu \mathrm{m}$ when subjected to tension-tension fatigue tests. Hofbeck et al. ${ }^{12)}$ also showed that the number of cycles to failure of copper wires with $30-90 \mu \mathrm{m}$ increases with a decrease in the diameter.

On the other hand, an inverse size effect has also been reported for fatigue behavior in the same size regime. Yang et $a l .{ }^{13)}$ showed that the fatigue life of copper wire decreases with a decrease in their diameter from 50 to $30 \mu \mathrm{m}$. Weiss et $a l .{ }^{14)}$ and Khatibi et al. ${ }^{15)}$ also obtained similar results in the diameter range of 10 to $125 \mu \mathrm{m}$. Similarly, Dai et al. ${ }^{16,17)}$ reported that fatigue resistance of copper foils decreases with a decrease in the foil thickness in the range from 20 to $420 \mu \mathrm{m}$. On the basis of the fact that if grain size in a foil remains unchanged, the number of grain boundaries decreases with a decrease in the foil thickness, they explained the obtained results in terms of the crack growth resistance of the grain boundaries. We note that they pointed out the influence of the crystallographic texture on fatigue life. Hatano et al. $^{1)}$ also reported that the copper foils with a welldeveloped cube texture showed superior fatigue lives.

Thus, to elucidate the intrinsic size effect on fatigue behavior on the micrometer scale, it is necessary to distinguish how the grain boundary and crystallographic orientation affect fatigue behavior. With this aim, in this study, we investigate how the crystallographic orientation and slip geometry contribute to the thickness effect on the fatigue behavior of the foils. We employed copper single-crystalline foils that had two different surface orientations and thicknesses of $140-500 \mu \mathrm{m}$.

\section{Experimental Procedure}

\subsection{Materials}

$\mathrm{Cu}$ single crystals with $(21 \overline{1})$ and $(5 \overline{1} \overline{1})$ surfaces were grown by the Bridgman method. The reason why these two mutually perpendicular surface orientations were chosen will 


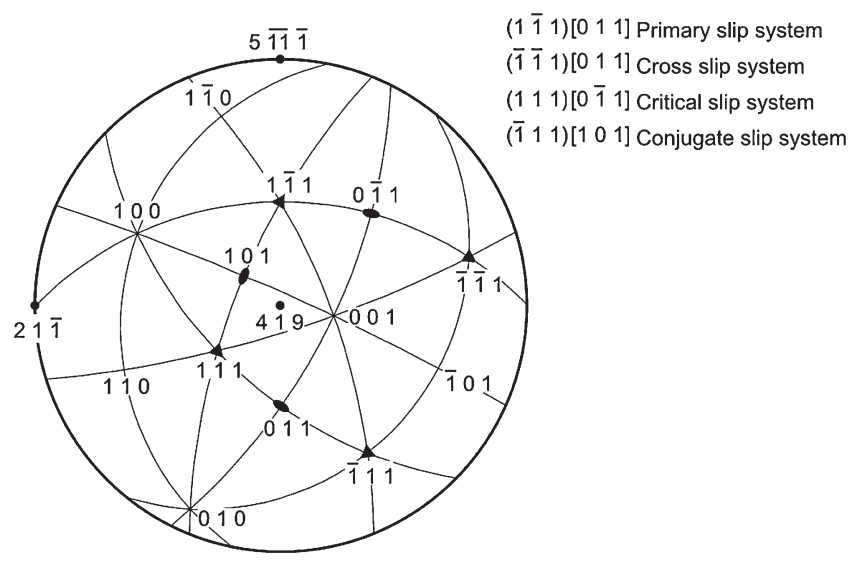

Fig. 1 Stereographic projection indicating the orientations of specimen surfaces, stress axis and four types of slip systems in the copper foil specimen. (a)

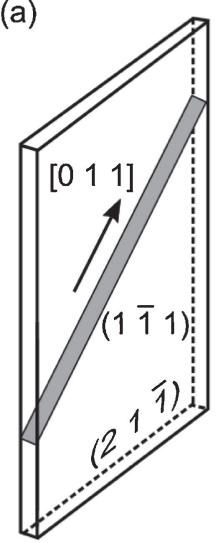

(b)

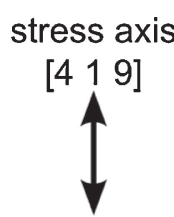

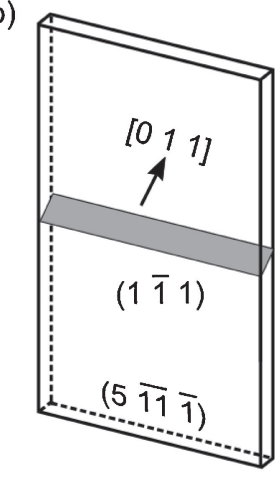

Fig. 2 Geometry of the primary slip plane in (a) the (21 $\overline{1})$ and (b) $(5 \overline{11} \overline{1})$ specimens.

be mentioned just below. Specimens with dimensions of $32 \mathrm{~mm} \times 6 \mathrm{~mm} \times 1 \mathrm{~mm}$ were cut from the single crystals by using an electric discharge machine so that the longitudinal axis becomes parallel to the [419] single-slip direction. It should be noted that [419] lies on both $(21 \overline{1})$ and $(5 \overline{11} \overline{1})$. The fatigue specimens were thinned down to $500 \mu \mathrm{m}, 300 \mu \mathrm{m}$ and $140 \mu \mathrm{m}$ by mechanical- and electro-polishing. Figure 1 shows the stereographic projection indicating the four possible slip systems. When the stress axis is [419], the Schmid factor of the (111)[011] primary slip system has the maximum value of 0.5 . Figure 2 shows geometrical relations among the stress axis, surface orientation and primary slip system for the $(21 \overline{1})$ and $(5 \overline{11} \overline{1})$ specimens. Both specimens have the same stress axis and therefore the same active primary slip system but distinctively different surface planes.

\subsection{Fatigue tests}

Shear stress in the primary slip system $\tau$ can be obtained as $0.5 F / a t$, where $F$ is the applied load, $a$ the width and $t$ the thickness of the specimen, respectively. Load-controlled tension-tension fatigue tests with stress ratio $R=\tau_{\min } /$ $\tau_{\max }=0$ were conducted at room temperature by using a magnetic micro testing machine (SHIMADZU MMT$500 \mathrm{NB}$ ) under various shear stress amplitudes $\tau_{\mathrm{c}}$ in the range of $33 \mathrm{MPa}$ to $55 \mathrm{MPa}$. Tension-tension fatigue test was (a)

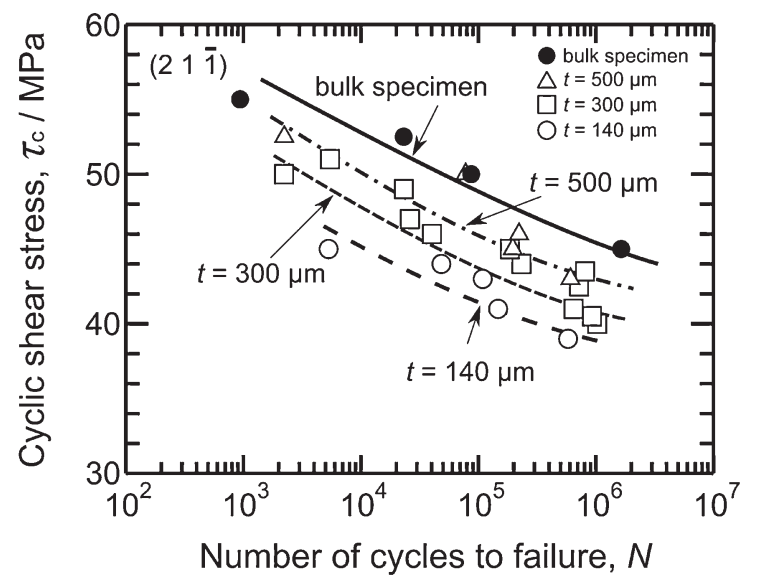

(b)

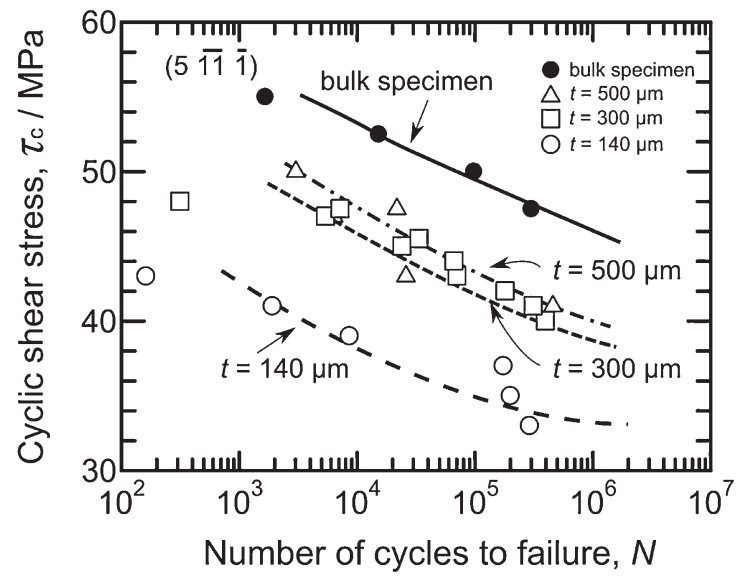

Fig. $3 S-N$ curves (fatigue life versus applied share stress amplitude) of (a) the $(21 \overline{1})$ and (b) (511) specimens with a thickness ranging from 140 to $500 \mu \mathrm{m}$.

selected to avoid buckling and deflecting during the fatigue tests. A triangular waveform signal with a frequency of $3.5 \mathrm{~Hz}$ was used.

\subsection{Microstructural characterization}

Specimen surfaces and fracture surfaces were observed by a field emission scanning electron microscope (FE-SEM, JSM-7001F). To reveal surface steps on the surface of the $(21 \overline{1})$ and $(5 \overline{11} \overline{1})$ specimens, cross-sectional samples were prepared by a focused ion beam milling technique, and then they were observed by SEM. Development of dislocation microstructure was also investigated using the (21) fatigued specimen by the electron channeling contrast imaging (ECCI) technique in FE-SEM.

\section{Results}

\section{1 $S$ - $N$ curves}

Figure 3(a) and (b) show $S-N$ curves of the (21) and $(5 \overline{11})$ specimens, respectively. The results of bulk specimens with a gauge dimension of $100 \mathrm{~mm} \times 2 \mathrm{~mm} \times 2 \mathrm{~mm}$ are also shown for comparison. It is found for both the $(21 \overline{1})$ and $(5 \overline{11} \overline{1})$ specimens that fatigue life decreases with decrease in foil thickness. Even if the thickness is as large as $500 \mu \mathrm{m}$, the fatigue life is shorter than that of the bulk specimen. It is notable that the $(5 \overline{11} \overline{1})$ specimens show a more pronounced thickness dependence of fatigue life than 

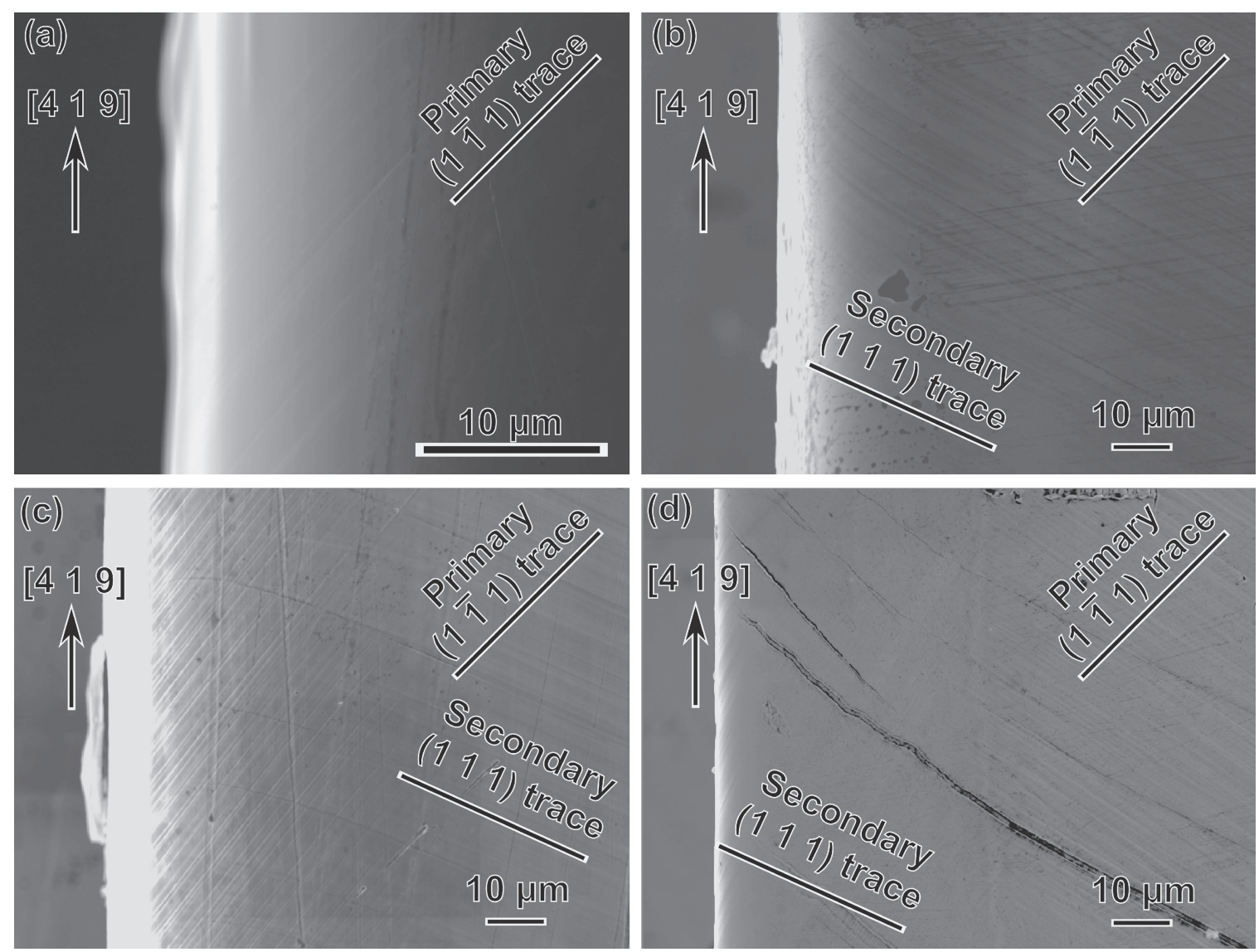

Fig. 4 SEM images of the (21) $)$ specimens with $300 \mu \mathrm{m}$ thickness fatigued to 5000 cycles under shear stress amplitude of (a) $10 \mathrm{MPa}$, (b) $20 \mathrm{MPa}$, (c) $30 \mathrm{MPa}$ and (d) $40 \mathrm{MPa}$, respectively. Only primary slip traces are seen in (a), whereas secondary slip traces are also visible in (b), (c), and (d) on the (21) $)$ surface.

the $(21 \overline{1})$ specimens. Furthermore, when we focus on the orientation dependence the fatigue life, for example, at the same specimen thickness of $140 \mu \mathrm{m}$, it can be seen that the fatigue life of the (21) specimen is about 1000 times longer than that of the $(5 \overline{11} \overline{1})$ specimen.

\subsection{Microstructural characterization}

Surfaces of the $300 \mu \mathrm{m}$-thick specimens fatigued to 5000 cycles under various stress amplitudes were observed by SEM. Figures 4 and 5 show the development of the slip lines

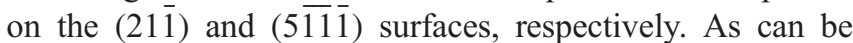
seen from Figs. 4(a) and 5(a), only the (11 1$)$ primary slip system was activated at a cyclic stress of $10 \mathrm{MPa}$. Since the [011] primary slip direction is parallel to the $(21 \overline{1})$ plane, the primary slip traces can barely be seen in Fig. 4(a). On the contrary, the primary slip traces and slip-related surface steps are clearly seen on the $(5 \overline{11} \overline{1})$ plane in Fig. 5(a). With increasing the stress amplitude from 10 to $40 \mathrm{MPa}$, the secondary slip traces parallel to the (111) plane become visible in Figs. 4 and 5. Two sets of slip steps parallel to the (1iㅣ) and (111) planes were well developed in the $(5 \overline{11} \overline{1})$ specimen fatigued at a stress amplitude of $40 \mathrm{MPa}$, as shown in Fig. 5(d). The cross-sectional image of the (511 $\overline{1})$ specimen fatigued at a stress amplitude of $44 \mathrm{MPa}$ for 66,000 cycles is shown in Fig. 6. Steps with a few micrometers height were formed along the (1) 1$)$ primary slip plane were formed due to the tension-tension loading mode in the present study. This fact also implies that the specimen is in a three-dimensional stress state during fatigue loading, while the specimen thickness is very small.

Figures 7(a) and (b) show the fracture surface of the (21. and $(5 \overline{11} \overline{1})$ specimens, respectively. The overall fracture plane of the (21 $\overline{1})$ specimen was almost parallel to the (111) secondary slip plane, and therefore a straightforward fracture was observed. The crack surface in the $(5 \overline{1} \overline{1})$ specimen, on the other hand, showed a complicated pattern formed by both the primary and secondary slips.

Figure 8 shows dislocation microstructure of the (21ㄷ) specimen with a $300 \mu \mathrm{m}$ thickness observed by the ECCI technique from the [21ㄱ] direction. Ladder-like dislocation wall structure parallel to the primary slip plane and cell structure were developed. No typical vein structure ${ }^{18-20)}$ was found in the matrix.

\section{Discussion}

It was found in the present study that thinner foils have lower fatigue lives in both the $(21 \overline{1})$ and $(5 \overline{1} \overline{1})$ specimens. Since only a small number of cycles are needed for crack propagation in the thin foil specimens, the crack initiation and the size of initial cracks are likely to be a dominant factor of fatigue life in the foils. Taking the above point into account, the reason why the fatigue life has such a strong thickness and orientation dependence will be discussed as follows.

Geometrical relationship among the primary (1히) slip plane, the foil surface, and the surface steps is given as 

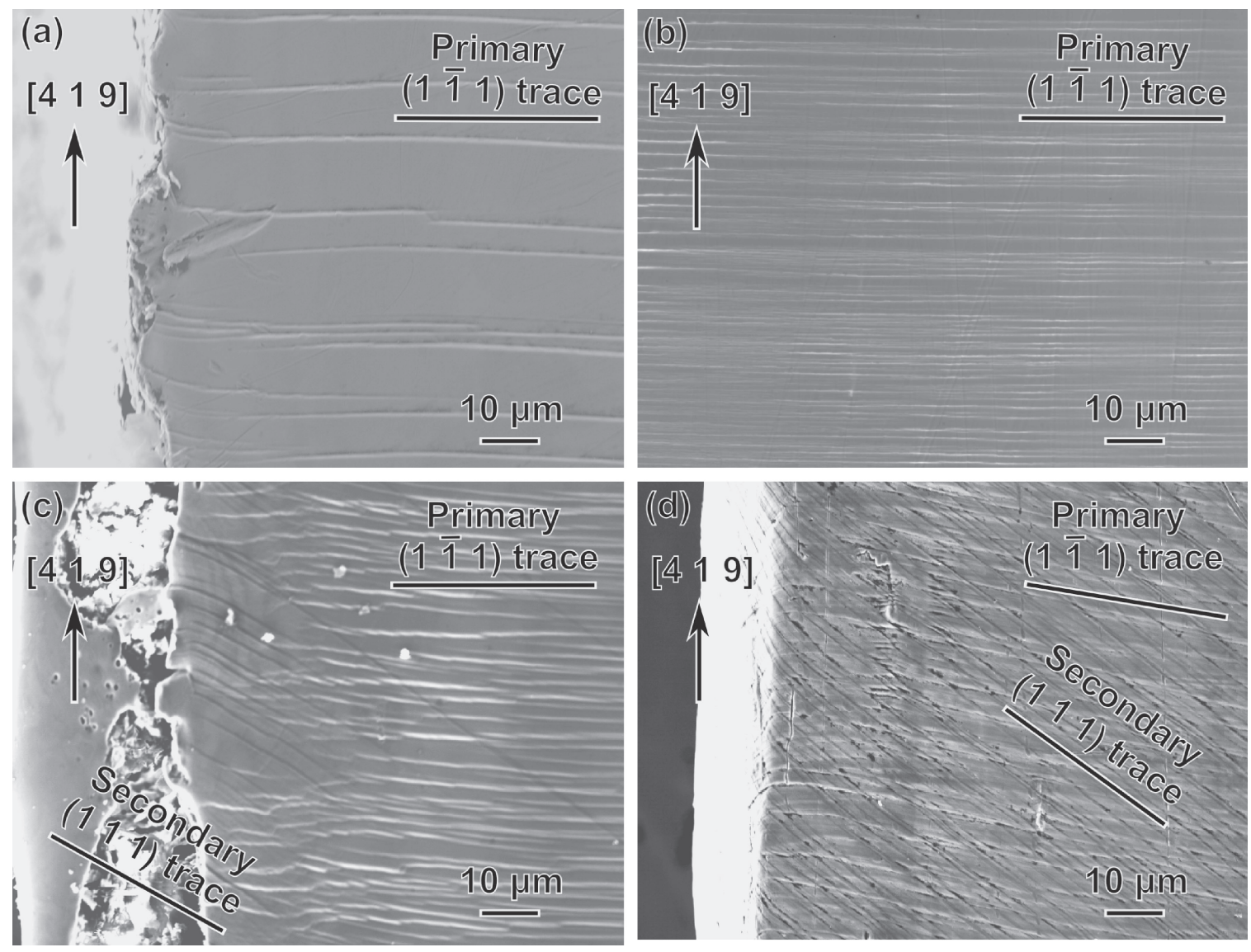

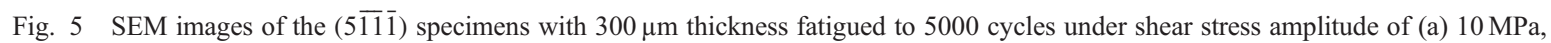
(b) $20 \mathrm{MPa}$, (c) $30 \mathrm{MPa}$ and (d) $40 \mathrm{MPa}$, respectively. Only primary slip traces are seen in (a), whereas secondary slip traces are also visible in (b), (c), and (d) on the (511 $\overline{1})$ surface.
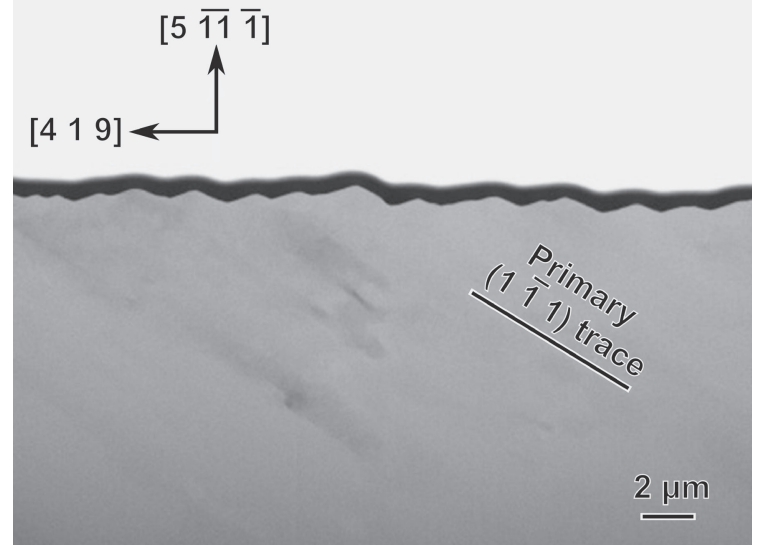

Fig. 6 A cross-sectional SEM image of the (5i11) specimen fatigued at a cyclic shear stress amplitude of $44 \mathrm{MPa}$ to 66,000 cycles. The specimen was observed from the [211̄] direction.

Fig. 9. For the two distinct specimens, i.e. the (21) and

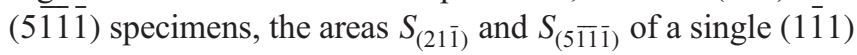
slip plane before deformation are simply calculated as

$$
S_{(21 \overline{1})}=S_{(5 \overline{11} \overline{1})}=\sqrt{2} a t,
$$

where $a$ and $t$ are respectively the width and length of the specimen. If we assume that the same amount of slip occurs at the primary slip plane in the two specimens, a step with a length $h$ is generated along the primary slip plane, as shown in Fig. 9. In the following analysis, lattice rotation due to deformation is not taken into account. After the deformation, the net areas of the primary slip plane in the specimens become, respectively,

$$
\begin{aligned}
& S_{(21 \overline{1})}^{\prime}=\sqrt{2} a t-h t, \\
& S_{(5 \overline{11} \overline{1})}^{\prime}=\sqrt{2} a t-h a .
\end{aligned}
$$

Therefore, the ratios $S_{(21 \overline{1})}^{\prime} / S_{(21 \overline{1})}$ and $S_{(5 \overline{1} \overline{1})}^{\prime} / S_{(5 \overline{11} \overline{1})}$ are expressed as

$$
\begin{aligned}
& \frac{S_{(21 \overline{1})}^{\prime}}{S_{(21 \overline{1})}}=1-\frac{1}{\sqrt{2}} \cdot \frac{h}{a}, \\
& \frac{S_{(5 \overline{1} \overline{1})}^{\prime}}{S_{(5 \overline{1} \overline{1})}}=1-\frac{1}{\sqrt{2}} \cdot \frac{h}{t} .
\end{aligned}
$$

We note here that although $S_{(21 \overline{1})}^{\prime} / S_{(21 \overline{1})}$ is a function independent of $t, S_{(5 \overline{1} \overline{1})}^{\prime} / S_{(5 \overline{11} \overline{1})}$ depends on the thickness $t$ and decreases with decreasing $t$. This fact implies that, if cyclic deformation is carried by only the primary slip system, the deformation of the $(5 \overline{11} \overline{1})$ specimen is significantly affected by the formation of surface steps and resultantly shows the thickness dependence of fatigue life. Therefore, the strong thickness dependence of the $S-N$ curves shown in Fig. 3(b) for the (5 $\overline{11} \overline{1})$ specimen can be simply explained by the above considerations. On the other hand, eq. (4) indicates that the fatigue behavior of the (21) specimen should be independent of the foil thickness if only the primary slip system is operative. In reality, however, the $S-N$ curves of the $(21 \overline{1})$ specimen shown in Fig. 3(a) depict the weak thickness dependence. To understand this phenomenon we will consider the activation of the secondary slip system by 

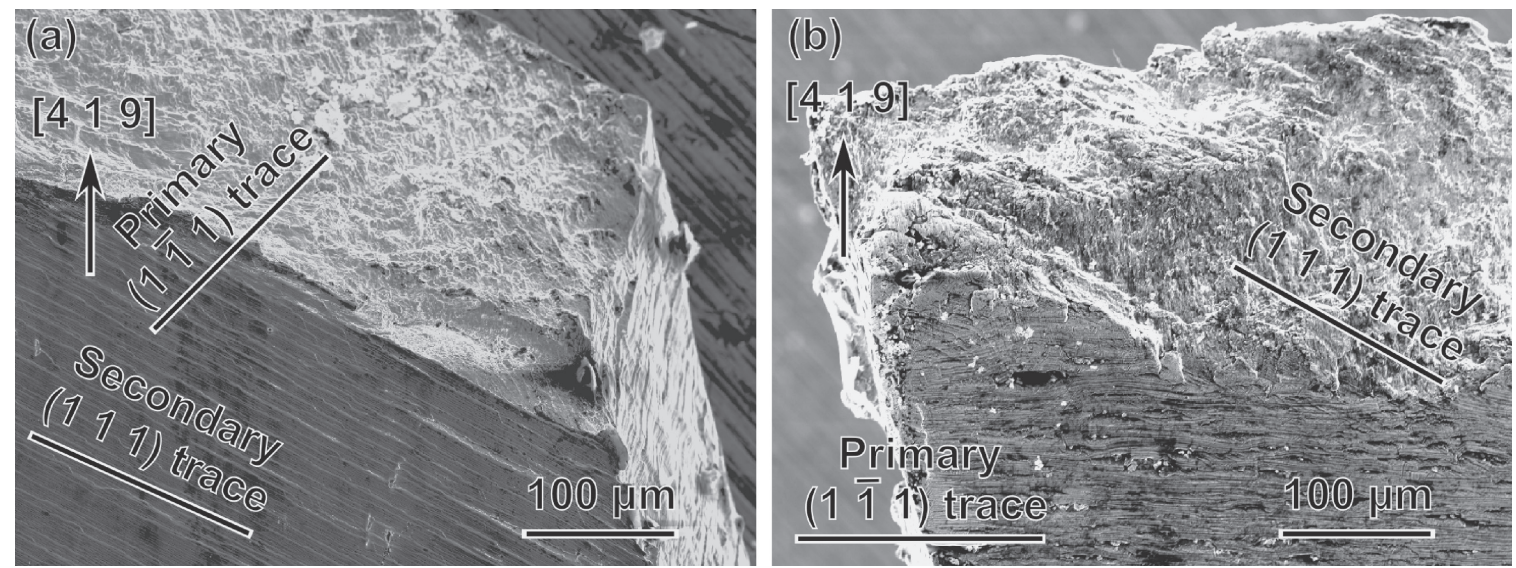

Fig. 7 SEM images of (a) the fractured (21) specimen fatigued to 2220 cycles and (b) the fractured (5i11) specimen fatigued to 28 cycles under a stress amplitude of $50 \mathrm{MPa}$. Foil thickness: $300 \mu \mathrm{m}$.

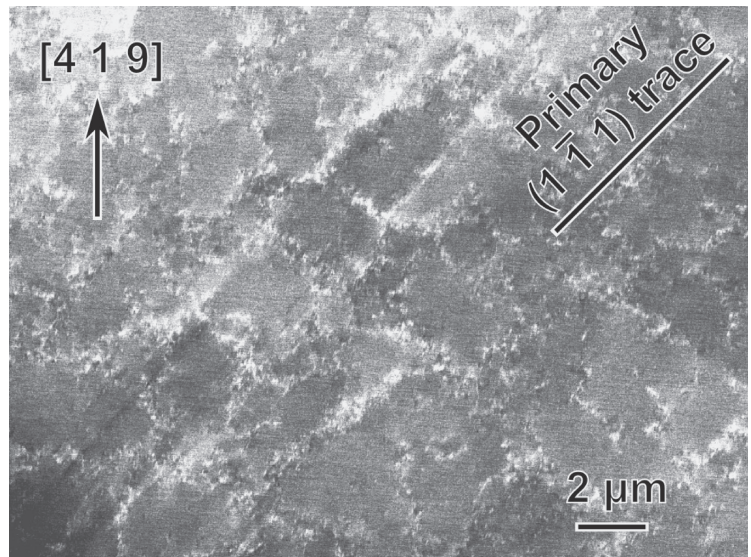

Fig. 8 Dislocation structure of the $(21 \overline{1})$ specimen fatigued at a cyclic shear stress amplitude of $28 \mathrm{MPa}$ to 5000 cycles. The specimen was observed from the [211] direction by using the ECCI technique. Foil thickness: $300 \mu \mathrm{m}$. (a)

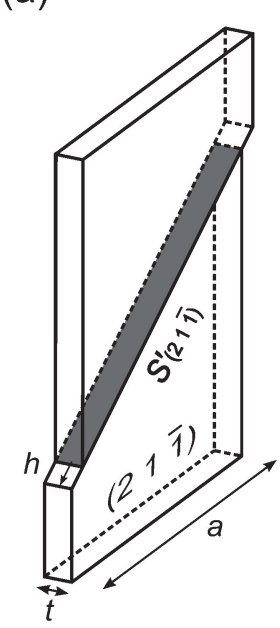

$\begin{array}{cc}\text { stress axis } & \text { (b) } \\ {\left[\begin{array}{lll}4 & 1 & 9\end{array}\right]} & \end{array}$

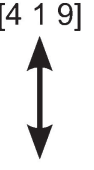

$S^{\prime}(5111)$

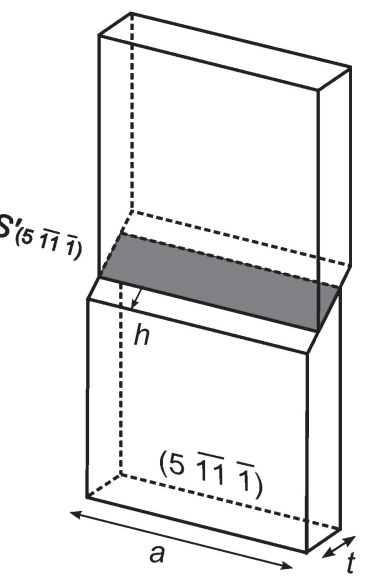

Fig. 9 Step formation caused by the primary slip in (a) the (21) and

(b) (511) specimens, where $a$ is the width of the specimen, $t$ the thickness, $h$ the displacement along the primary slip direction, and $S^{\prime}$ the area of the primary slip plane after deformation.

noting the results shown in Figs. 4 and 5. For the (211) and $(5 \overline{11} \overline{1})$ specimens before deformation, the areas $S_{\mathrm{s}(21 \overline{1})}$ and $S_{\mathrm{s}(5 \overline{1} \overline{1})}$ of a secondary (111) slip plane are calculated as (a)

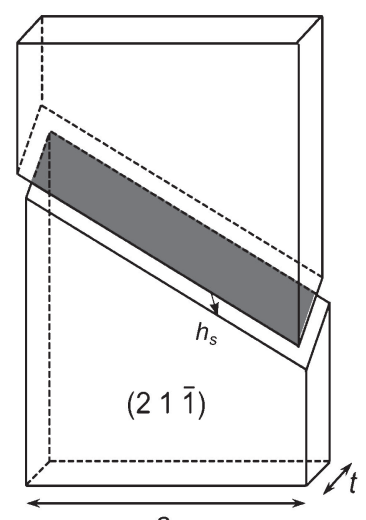

stress axis

[4 19$]$

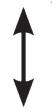

$(5 \overline{11} \overline{1})$

(b)

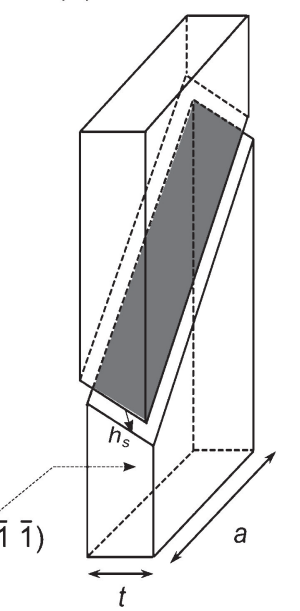

Fig. 10 Step formation caused by the secondary slip in (a) the (21ㅅ) and (b) $(5 \overline{11})$ specimens, where $h_{\mathrm{s}}$ is the displacement along the secondary slip direction.

$$
S_{\mathrm{s}(21 \overline{1})}=S_{\mathrm{s}(5 \overline{1} \overline{1})}=\frac{3}{\sqrt{6}} \cdot a t .
$$

As was the case of the primary slip, a step with a length $h_{\mathrm{s}}$ is generated along the secondary slip direction when the secondary slip system is activated in the respective specimens as shown in Fig. 10. After the deformation, the net areas of the secondary slip plane in the specimens become

$$
\begin{aligned}
& S_{\mathrm{s}(211)}^{\prime}=\frac{3}{\sqrt{6}} \cdot a t-\frac{5}{7} \cdot h_{\mathrm{s}} t-\frac{1}{\sqrt{2}} \cdot h_{\mathrm{s}} a+\frac{5}{7 \sqrt{3}} \cdot h_{\mathrm{s}}{ }^{2}, \\
& S_{\mathrm{s}(5 \overline{1} \overline{1})}^{\prime}=\frac{3}{\sqrt{6}} \cdot a t-\frac{1}{\sqrt{2}} \cdot h_{\mathrm{s}} t-\frac{5}{7} \cdot h_{\mathrm{s}} a+\frac{5}{7 \sqrt{3}} \cdot h_{\mathrm{s}}{ }^{2} .
\end{aligned}
$$

Then the ratios $S_{\mathrm{s}(21 \overline{1})}^{\prime} / S_{\mathrm{s}(21 \overline{1})}$ and $S_{\mathrm{s}(5 \overline{1} \overline{1})}^{\prime} / S_{\mathrm{s}(5 \overline{1} \overline{1})}$ are derived as

$$
\begin{aligned}
& \frac{S_{\mathrm{s}(21 \overline{1})}^{\prime}}{S_{\mathrm{s}(21 \overline{1})}}=1-\frac{5 \sqrt{6}}{21} \cdot \frac{h_{\mathrm{s}}}{a}-\frac{\sqrt{3}}{3} \cdot \frac{h_{\mathrm{s}}}{t}+\frac{5 \sqrt{2}}{21} \cdot \frac{h_{\mathrm{s}}{ }^{2}}{a t}, \\
& \frac{S_{\mathrm{s}(5 \overline{1} \overline{1})}^{\prime}}{S_{\mathrm{s}(511 \overline{1})}}=1-\frac{\sqrt{3}}{3} \cdot \frac{h_{\mathrm{s}}}{a}-\frac{5 \sqrt{6}}{21} \cdot \frac{h_{\mathrm{s}}}{t}+\frac{5 \sqrt{2}}{21} \cdot \frac{h_{\mathrm{s}}{ }^{2}}{a t} .
\end{aligned}
$$

It is easy to see that both the ratios decrease with decreasing $t$. This leads to the conclusion that the $S-N$ curves have the thickness dependence not only in the $(5 \overline{11} \overline{1})$ specimen but also in the (211) specimen. Thus the weak thickness 


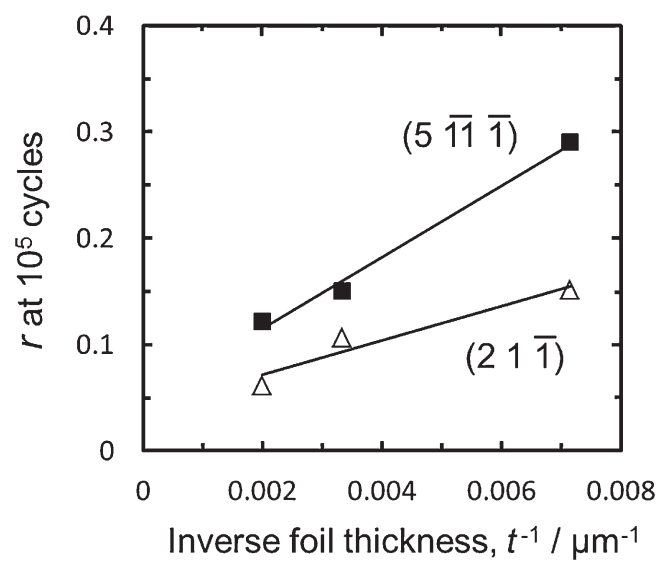

Fig. 11 The relationship between $r$ and $1 / t$. See the text for the definition of $r$.

dependence of the $S-N$ curves in the (21) specimen can be explained by considering the effects of the secondary slip.

To confirm the above interpretation of the effects of the crystallography and slip geometry on the thickness dependence of the fatigue life, we now examine whether or not the thickness dependence of failure stress amplitudes in the $S$ $N$ curves can be quantitatively explained by a reduction of the slip plane. We define a ratio $r$, derived from the shear stress amplitude $\tau_{\mathrm{B}}$ for the bulk specimen and $\tau_{\mathrm{F}}$ for the foil specimens where the cycle of failure become $10^{5}$, as

$$
r=\frac{\left|\tau_{\mathrm{F}}-\tau_{\mathrm{B}}\right|}{\tau_{\mathrm{B}}} .
$$

Figure 11 shows the relationship between $r$ and $1 / t$ for the $(21 \overline{1})$ and $(5 \overline{1} \overline{1})$ specimens. It can be seen that there exists a linear relationship between $r$ and $1 / t$ for each specimen. The slope of the line for the $(5 \overline{11} \overline{1})$ specimen is about twice that of the line for the $(21 \overline{1})$ specimen. This means that the $(5 \overline{11} \overline{1})$ specimen shows stronger size dependence of the fatigue life than the $(21 \overline{1})$ specimen. By using eqs. (5) and (10) for the $(5 \overline{11} \overline{1})$ specimen, where $h / a, h_{\mathrm{s}} / a$ and $h_{\mathrm{s}}^{2} / a t$ are assumed to be negligibly small compared with $h / t$ and $h_{\mathrm{s}} / t$, the theoretical slope of the $(5 \overline{1} \overline{1})$ straight line becomes $0.71 h+0.58 h_{\mathrm{s}}$. Similarly, the theoretical slope for the (21) specimen in eq. (9) is $0.58 h_{\mathrm{s}}$. Then the ratio of the above two theoretical slopes is almost the same as the ratio of the experimental slopes in Fig. 11, when $h$ and $h_{\mathrm{s}}$ are assumed to be the same (a few micrometers). Therefore, it is reasonably understood that the slip geometry is a dominant factor of the size effect of the fatigue life in single-crystalline foils.

In this study, we treated the single crystal foils with a thickness range of $140 \mu \mathrm{m}$ to $500 \mu \mathrm{m}$ and conducted tensiontension cyclic fatigue tests. Since the heights of the developed surface steps were in the micrometer range, the slip-plane reduction effects can be ignored in the bulk specimen. On the other hand, if the specimen thickness decreases to a few micrometers, it seems to be hard to develop steps with a few micrometers in dimension. Another mechanism, such as a dislocation starvation, ${ }^{5)}$ may be responsible for explaining the size effects on fatigue behavior of the very thin foils. As mentioned in Section 1, the controversial results about the size effects on fatigue life were reported in previous literature. This is caused partly by a lack of consideration of the crystallographic orientation of the specimen surfaces. The present study using single-crystalline foils clearly shows that the surface orientation is one of the key factors to affect the fatigue life of thin foils with a thickness of several hundred microns.

\section{Conclusions}

Cyclic load-controlled tension-tension fatigue tests of

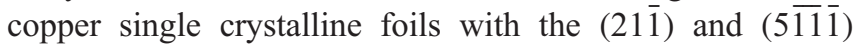
surface orientations were carried out for the thickness ranging from 140 to $500 \mu \mathrm{m}$. The following conclusions are drawn from the $S-N$ curves and microstructural observation.

(1) Both the (21) and $(5 \overline{11} \overline{1})$ specimens within given range of foil thickness show the thickness dependence of fatigue life, i.e. fatigue life becomes shorter with decreasing the foil thickness.

(2) Thickness dependence of the $(5 \overline{1} \overline{1})$ specimen is more pronounced than that of the $(21 \overline{1})$ specimen.

(3) When the thicknesses of the two types of foils are the same, fatigue life of the (21) specimen is longer (by a factor of about 1000$)$ than that of the $(5 \overline{11} \overline{1})$ specimen.

(4) Ladder-like dislocation wall structure is developed in the foil with a $300 \mu \mathrm{m}$ thickness.

(5) The observed effects of specimen thickness and orientation on the fatigue life of copper single crystalline foils can be explained by a simple slip model based on the geometrical relationship between the foil surface and the primary and secondary slip systems.

\section{REFERENCES}

1) T. Hatano, Y. Kurosawa and J. Miyake: J. Electron. Mater. 29 (2000) $611-616$.

2) W. Engelmaier and A. Wagner: Circuit World 14 (1988) 30-38.

3) W. D. Nix: Metall. Trans. A 20 (1989) 2217-2245.

4) E. Arzt: Acta Mater. 46 (1998) 5611-5626.

5) J. R. Greer and W. D. Nix: Appl. Phys. A 80 (2005) 1625-1629.

6) C. Motz, T. Schöerl and R. Pippan: Acta Mater. 53 (2005) 4269-4279.

7) M. Hommel and O. Kraft: Acta Mater. 49 (2001) 3935-3947.

8) O. Kraft, P. Wellner, M. Hommel, R. Schwaiger and E. Arzt: Z. Metallkd. 93 (2002) 392-400.

9) G. P. Zhang, C. A. Volkert, R. Schwaiger, P. Wellner, E. Arzt and O. Kraft: Acta Mater. 54 (2006) 3127-3139.

10) G. P. Zhang, R. Schwaiger, C. A. Volkert and O. Kraft: Philos. Mag. Lett. 83 (2003) 477-483.

11) M. Judelewicz, H. U. Künzi, N. Merk and B. Ilschner: Mater. Sci. Eng. A 186 (1994) 135-142.

12) R. Hofbeck, K. Hausmann, B. Ilschner and H. U. Künzi: Scr. Metall. 20 (1986) 1601-1605.

13) B. Yang, C. Motz, W. Grosinger and G. Dehm: Mater. Sci. Eng. A 515 (2009) 71-78.

14) B. Weiss, V. Gröger, G. Khatibi, A. Kotas, P. Zimprich, R. Stickler and B. Zagar: Sensor Actuator A Phys. 99 (2002) 172-182.

15) G. Khatibi, A. Betzwar-kotas, V. Groger and B. Weiss: Fatigue Fract. Eng. Mater. Struct. 28 (2005) 723-733.

16) C. Y. Dai, G. P. Zhang and C. Yan: Philos. Mag. 91 (2011) 932-945.

17) C. Y. Dai, B. Zhang, J. Xu and G. P. Zhang: Mater. Sci. Eng. A 575 (2013) 217-222.

18) A. T. Winter: Philos. Mag. 30 (1974) 719-738.

19) Z. S. Basinski and S. J. Basinski: Prog. Mater. Sci. 36 (1992) 89-148.

20) P. Li, S. X. Li, Z. G. Wang and Z. F. Zhang: Prog. Mater. Sci. 56 (2011) $328-377$. 